\title{
Effects of Forming Conditions on Wrinkling in Necking of Tube End*1
}

\author{
Yoichi Takahashi $^{1}$, Shigefumi Kihara ${ }^{1}$, Takuo Nagamachi ${ }^{2}$, Hiroaki Mizumoto ${ }^{3}$ and Yuya Nakata ${ }^{4, * 2}$ \\ ${ }^{1}$ Department of Mechanical Engineering, Kagawa National College of Technology, Takamatsu 761-8058, Japan \\ ${ }^{2}$ Institute of Technology and Science, The University of Tokushima, Tokushima 770-8506, Japan \\ ${ }^{3}$ Nihon Spindle MFG. Co., Ltd., Amagasaki 661-8510, Japan \\ ${ }^{4}$ Advanced Course, Kagawa National College of Technology, Takamatsu 761-8058, Japan
}

\begin{abstract}
The effects of forming conditions on wrinkling in the neck of stainless-steel tubes were investigated using experiments and threedimensional finite element simulations. The materials used for this study were tubes designed for automotive exhaust parts. When the axial or radial forming pitch of the rollers is large, the outside of the tube is subjected to considerable compressive stress in the circumferential direction by contact with the rollers. Simultaneously, the inside of the tube is subjected to considerable tensile stress in the circumferential direction. Consequently, the tube is subjected to excessive localized forming, which causes the curvature to become uneven in the circumferential direction, thereby resulting in wrinkling. The number of contact points with the rollers was determined. Results show that the optimum forming conditions were easily determined to achieve short forming times and high dimensional accuracy. [doi:10.2320/matertrans.P-M2010822]
\end{abstract}

(Received May 13, 2010; Accepted August 27, 2010; Published December 8, 2010)

Keywords: rotary forming, tube forming, necking, explicit finite element method, wrinkle

\section{Introduction}

Necking is a rotary forming method for reducing the diameter of a tube at the center and ends by applying external force. ${ }^{1)}$ It has been used for fabrication of cylindrical products for automotive exhaust parts and home electronic appliances. Recently, diversified small-quantity production has become necessary to meet demands for increasingly diversified products by necking processes. Furthermore, reduction of manufacturing cost has been strongly desired. ${ }^{2,3)}$ The force on rollers used for the necking is much smaller than that on tools used for conventional press forming processes. Therefore, it is possible to reduce the size of roller and equipment used for the necking, thus reducing the manufacturing cost. Because of these advantages, the necking has come to attract attention as a tube forming technique that can readily meet the requirements for low-cost manufacturing and a diversified small-quantity production.

During the necking tubes deform in a complicated manner because they have a large free surface that has no contact with the rollers. Moreover, it is difficult to determine the appropriate forming conditions that minimize the defects such as cracking and wrinkling because the necking process involves more factors affecting tube deformation than other forming methods involve. Consequently, to date, forming conditions and fabrication accuracy during necking processes have been investigated through experimental studies ${ }^{1,2,4)}$ and numerical simulations. ${ }^{5-7)}$ However, the deformation behavior still remains unclear, and the mechanism of wrinkling has not been clarified.

In this study, we conducted experiments and simulations to investigate the necking of car exhaust pipes. ${ }^{8,9)}$ The mechanisms underlying the material deformation and occurrence of wrinkling are discussed from the results. Additionally,

\footnotetext{
*1 This Paper was Originally Published in Japanese in the J. JSTP 51 (2010) 348-352.

${ }^{*}$ Undergraduate Student, Kagawa National College of Technology
}

the number of contacts with the rollers is defined, and the conditions of necking limit are discussed.

\section{Experimental and Simulation Methods}

Figure 1 show schematic illustrations of the necking process. The tube was $120.0 \mathrm{~mm}$ in outer diameter, $112.0 \mathrm{~mm}$ long, and $1.5 \mathrm{~mm}$ thick. The outer diameter was reduced to $54.0 \mathrm{~mm}$ using rollers having an outer diameter of $100.0 \mathrm{~mm}$ and radius of curvature of $8.0 \mathrm{~mm}$. The taper angle of the necking was $28.8^{\circ}$. The reduction ratio of the tube end was 0.45 . In this process, the rollers moved spirally around the axis of tube in the axial direction, as well as in radial directions. Figure 1(c) shows the locus of the tip of a roller in the $r-z$ plane. The axial feed rate and radial pitch of the rollers during necking are denoted, as $L$ and $P$, respectively. The rollers moved back and forward for $N$ passes and then only move forward at the final pass to achieve the desired outer diameter of the tube end. The total distance of the rollers in the radial direction from the start to the end of forming was $r_{\mathrm{P}}=33.0 \mathrm{~mm}$. The necking process comprises a total of $N=\operatorname{int}\left(r_{\mathrm{P}} / P\right)+1$ passes. Here, $\operatorname{int}(X)$ is an operation that gives the integer part of real number $X$. The number of rollers $N_{\mathrm{R}}$ used for the forming was $1-3$. Two and three rollers maintain an interval of $180^{\circ}$ and $120^{\circ}$ during necking as shown in Fig. 2. Therefore, the $L$ decreases as the $N_{\mathrm{R}}$ increases. The forming pitch in the axial direction can be given as $L / N_{\mathrm{R}}$.

Tables 1 and 2 present the forming conditions and the mechanical properties of the tube, respectively. Experiments were conducted in addition to a simulation under the condition of two rollers forming shown in Table 1. Simulations for only $N_{\mathrm{R}}=1$ and 3 were conducted. Tubes used for this study were made of ferrite stainless steel. The mechanical properties presented in Table 2 were determined from tensile tests on JIS Z 2201 13B specimens cut from these tubes.

A tube spinning machine (VF-SR150-CNC4-T4; Nihon Spindle Manufacturing Co., Ltd.) was used in the experi- 


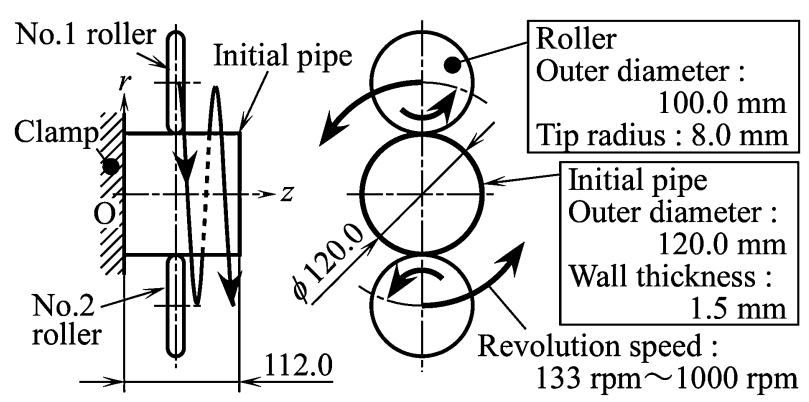

(a) Dimensions of roller and initial pipe

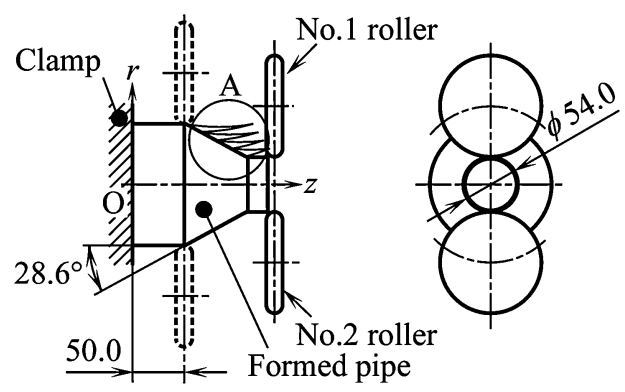

(b) Dimensions of finished pipe

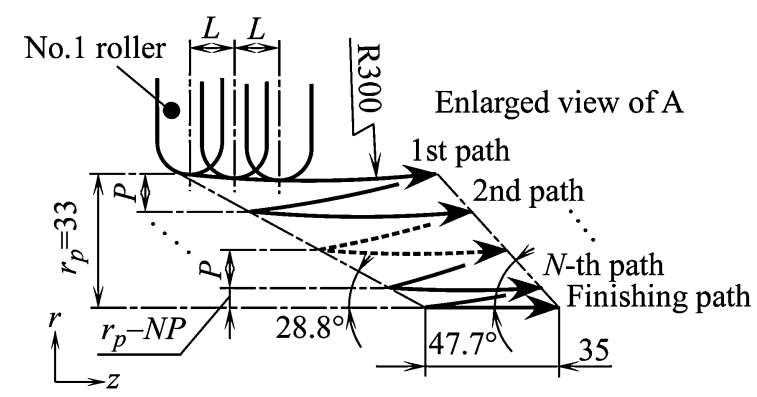

(c) Locus of No.1 roller in $r-z$ plane

Fig. 1 Schematic illustration of necking process.

ments. To examine the dimensional accuracy of the tube end that was subjected to necking, the cross section of the necked portion of the tube was measured and roundness $\Delta r$ was calculated using the Maximum-Inscribed-Circle (MIC) method. For the simulation, the dynamic explicit code RADIOSS (Altair Engineering Inc.) was used. The tube was modeled using 5520 hexahedral elements. Three elements were allocated in the direction of thickness. As a boundary condition, the clamped end in the experiment was assumed to be fully fixed. It was also assumed that the rollers were rigid and that the coefficient of friction between the tube and the rollers was 0.15 . In the simulation, the rollers rotate due to the contact with the tube. We compared the stresses of the tube calculated preliminarily by using the various values of material density and the ratios of kinetic energy to total energy obtained in the calculation. We also compared the longitudinal distribution of the tube thickness obtained in the simulation and with that obtained by experiment. Considering the results and the calculation efficiency, the material density was set to $7.8 \times 10^{-4} \mathrm{~kg} / \mathrm{mm}^{3}$ for the present simulations. The ratio of the kinetic energy to the total energy at this density was less than $1 \%$. The results demonstrated that the stress calculated using the method described above was equivalent to that calculated using the

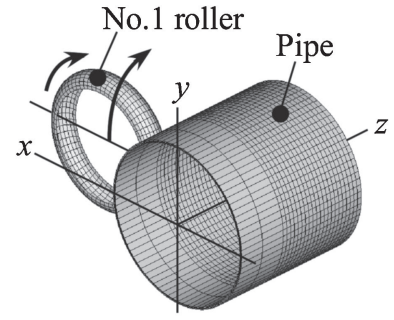

(a) Forming by one roller $\left(N_{R}=1\right)$

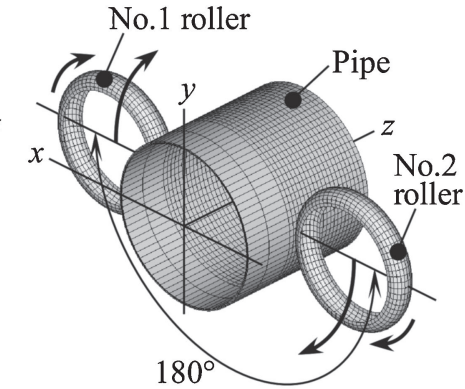

(b) Forming by two rollers $\left(N_{R}=2\right)$

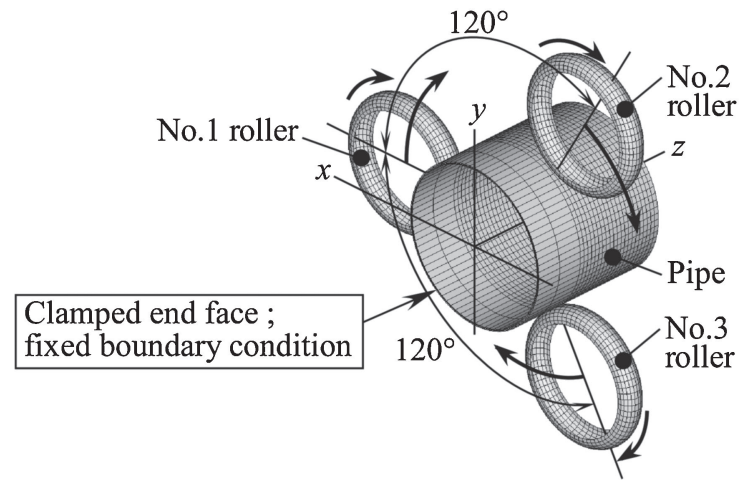

(c) Forming by three rollers $\left(N_{R}=3\right)$

Fig. 2 Initial model for finite element analysis.

Table 1 Forming conditions.

\begin{tabular}{ccc}
\hline $\begin{array}{c}\text { Number of } \\
\text { rollers } N_{\mathrm{R}}\end{array}$ & $\begin{array}{c}\text { Radial pitch } \\
P / \mathrm{mm}\end{array}$ & \multicolumn{1}{c}{$\begin{array}{c}\text { Axial feed rate } \\
L / \mathrm{mm}\end{array}$} \\
\hline 1 & 2.4 & $8.0,10.0,12.0,15.0$ \\
\hline & 1.6 & $6.0,12.0,24.0,30.0$ \\
\cline { 2 - 3 } 2 & 2.4 & $6.0,12.0,20.0,24.0,30.0$ \\
\cline { 2 - 3 } & 3.0 & $6.0,12.0,16.0,24.0$ \\
\cline { 2 - 3 } & 5.0 & $6.0,12.0,16.0$ \\
\hline 3 & 7.0 & $6.0,12.0,16.0$ \\
\hline
\end{tabular}

Table 2 Mechanical properties of initial pipe.

Young's modulus: $210.0 \mathrm{GPa}$
Poisson's ratio: 0.3
$0.2 \%$ proof stress: $230.0 \mathrm{MPa}$
$n$ value: 0.250
Flow stress: $\bar{\sigma}=280.0 \bar{\varepsilon}^{0.250}+110.0 \mathrm{MPa}$

static implicit method (implicit mode of RADIOSS). The calculation time was around $80 \mathrm{~h}$ for one condition (2 CPUs with $3.6 \mathrm{GHz}$ Xeon processors; Intel Corp.).

\section{Results and Discussion}

\subsection{Deformed shape after necking by two rollers}

Figure 3 shows the appearance of the tube after the experiment, in addition to simulation results obtained using the following parameters: $P=2.4 \mathrm{~mm}, L=6.0 \mathrm{~mm}$ and $P=$ 


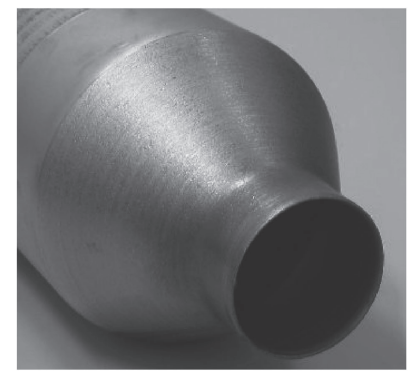

Experimental result

(a) $L=6.0 \mathrm{~mm}$

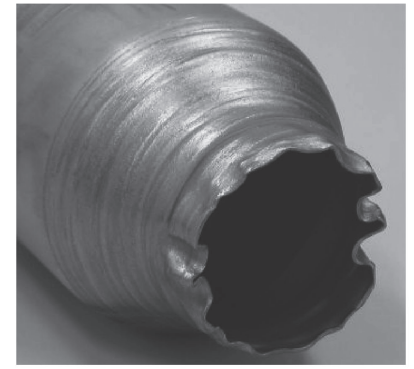

Experimental result

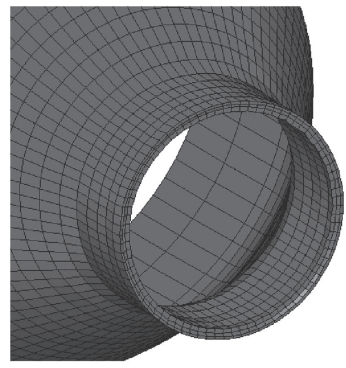

Simulation result

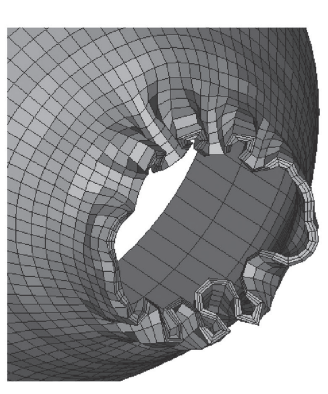

Simulation result

(b) $L=30.0 \mathrm{~mm}$

Fig. 3 Appearance of formed pipe $(P=2.4 \mathrm{~mm})$.

$2.4 \mathrm{~mm}, L=30.0 \mathrm{~mm}$. As observed in Fig. 3(a), the tube was formed with high dimensional accuracy in both the experiment and the simulation. The $\Delta r$ remained within $0.13 \mathrm{~mm}$ in the experiment and $0.14 \mathrm{~mm}$ in the simulation. For $L=$ $30.0 \mathrm{~mm}$, however, wrinkling occurred at the tube end during the first half of necking, at which point the radius of the necked portion of tube $r$ was reduced to $50 \mathrm{~mm}$. Hayama ${ }^{1)}$ reported that when the feed speed was low and the pitch is large in the same pitch necking experiment of the tube end, the reduction of thickness at the necked portion increases, thus causing cracking. Additionally, when the feed speed and pitch increase, wrinkling occurred at the tip of the tapered portion during tapering of the tube end. In this study, cracking did not occur in any forming condition in the experiments. With the condition of large $P$ and $L$, wrinkling occurred at the tube end. In the present paper, the occurrence of wrinkling is discussed by focusing on the deformation of the tube end when the $r$ is approximately $50 \mathrm{~mm}$ at the first half of the necking.

Figure 4(a) shows the distribution of curvature $\rho^{-1}$ in the circumferential direction at the reduced portion of the tube under the simulation condition of $P=7.0 \mathrm{~mm}$ and $L=$ $6.0 \mathrm{~mm}$. As shown in Fig. 4, the $\rho^{-1}$ is obtained at the three nodal coordinates on the outer surface of the cross section at a position of $z_{\mathrm{m}}=117.0 \mathrm{~mm}$, which is $4.0 \mathrm{~mm}$ apart from the tube end. The $\rho^{-1}$ shown in Fig. 4 is under the following conditions on the roller position: the coordinate of the tube end in the radial direction (target radius of the reduced tube), $r_{\mathrm{R}}=45.6 \mathrm{~mm}$; the coordinate in the longitudinal direction, $z_{\mathrm{R}}=112.6-115.0 \mathrm{~mm}$; and the angle of the axis center of the roller, $\alpha=36^{\circ}, 72^{\circ}, 108^{\circ}, 144^{\circ}$, and $180^{\circ}$. Focusing on the angular position of $\theta=72^{\circ}$, the $\rho^{-1}$ is $0.022 \mathrm{~mm}^{-1}$ at $\alpha=36^{\circ}$, whereas the $\rho^{-1}$ decreases to $0.016 \mathrm{~mm}^{-1}$ at
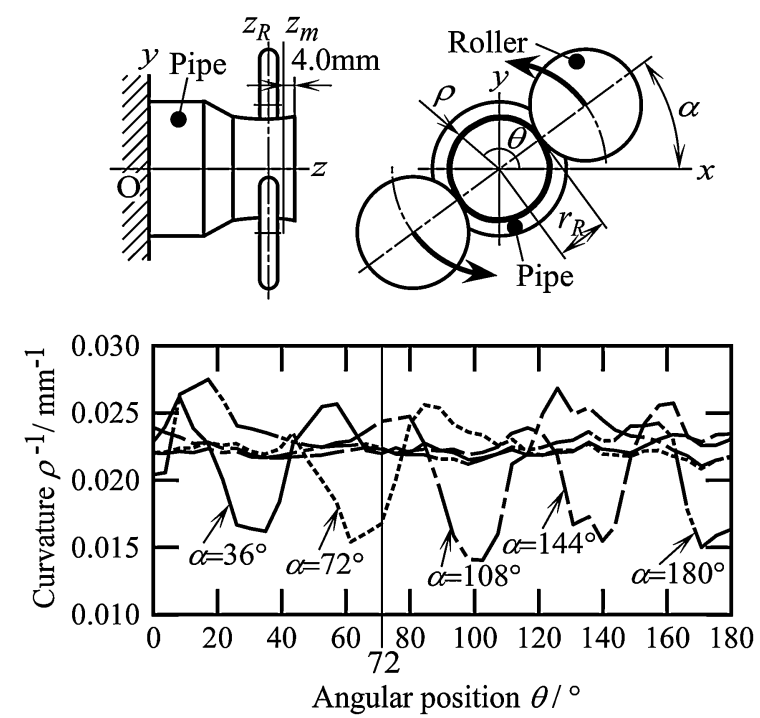

(a) $L=6.0 \mathrm{~mm}, z_{R}=112.6 \sim 115.0 \mathrm{~mm}$

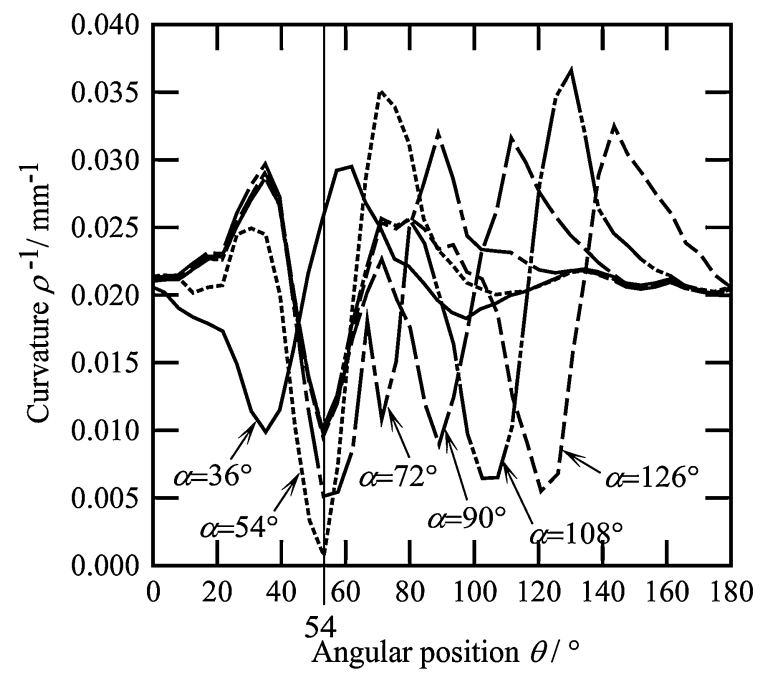

(b) $L=16.0 \mathrm{~mm}, z_{R}=110.0 \sim 115.0 \mathrm{~mm}$

Fig. 4 Transition of curvature distribution in cross section at $z=z_{\mathrm{m}}=$ $117 \mathrm{~mm}\left(P=7.0 \mathrm{~mm}, r_{\mathrm{R}}=45.6 \mathrm{~mm}\right.$, simulation result $)$.

$\alpha=72^{\circ}$ because of contact between the tube and the rollers. However, the $\rho^{-1}$ increases again to $0.024 \mathrm{~mm}^{-1}$ at $\alpha=108^{\circ}$. The $\rho^{-1}$ reaches approximately $0.022 \mathrm{~mm}^{-1}$ at $\alpha \geq 144^{\circ}$, which is the target curvature of the reduced tube. The reduction of diameter proceeds gradually through repeated unbending in which the curvature decreases such that the tube shape conforms to the geometry of roller due to the contact, and bending in which the curvature increases to maintain the shape of closed cross section of the tube end after separation from the roller. Takizawa et al., ${ }^{10)}$ based on finite element analysis and experiment on helical rolling of tube, reported that the direction of bending deformation in the circumferential direction changes before and after contact with the roller, and that such deformation is a redundant deformation. In the present study, the similar unbending and bending deformations are repeated. Under the conditions shown in Fig. 4(a), no excess unbending deformation remains after the forming is finished. The actual $\Delta r$ at the necked portion of the tube under the conditions described above is $0.12 \mathrm{~mm}$, which indicates that the necking was accomplished with high dimensional accuracy. Figure 4(b) 

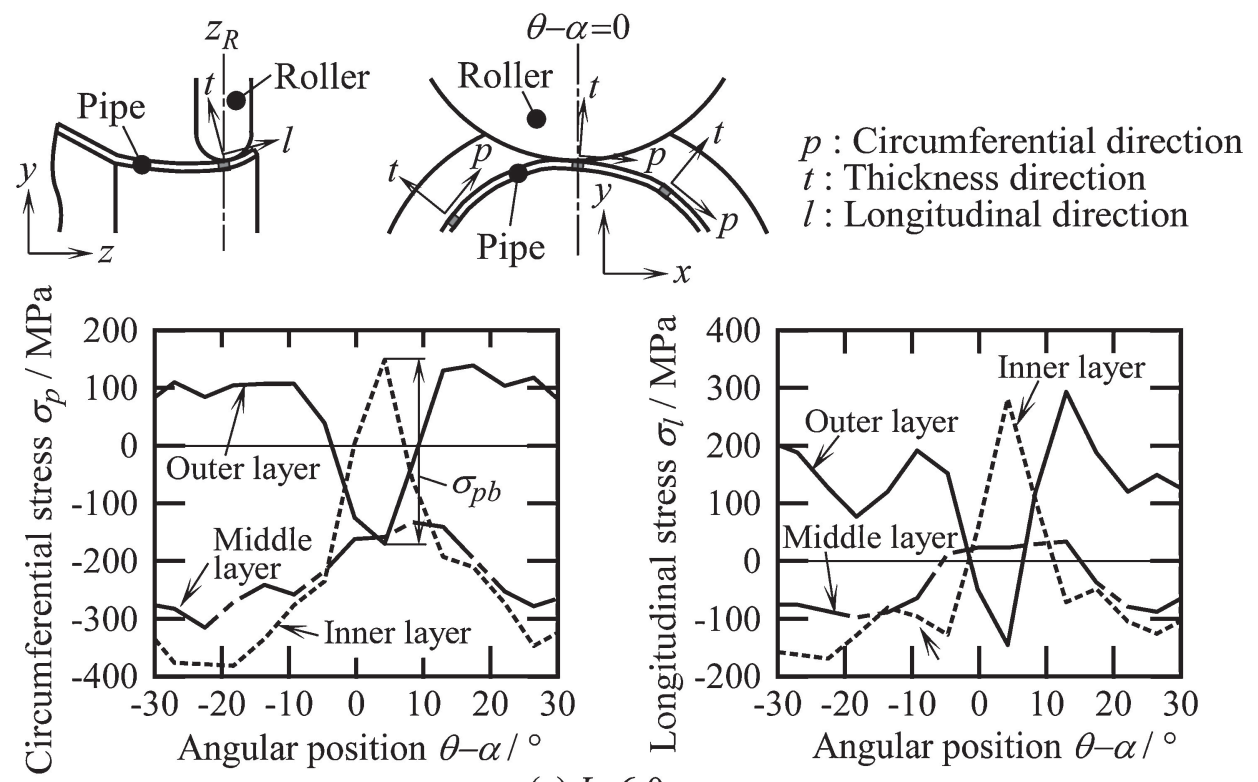

(a) $L=6.0 \mathrm{~mm}$
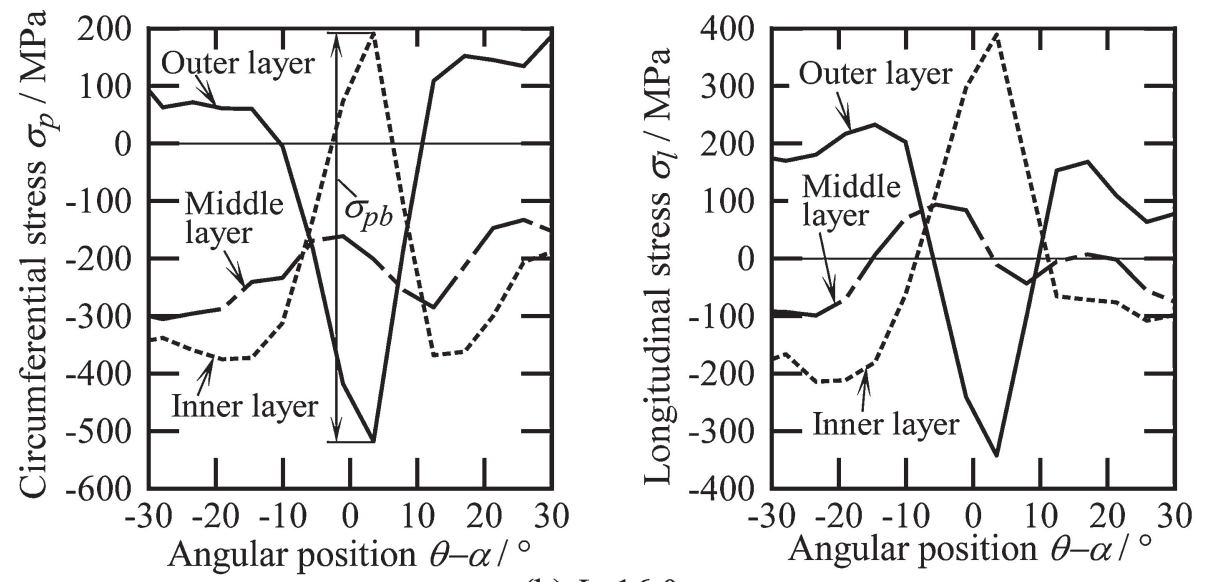

(b) $L=16.0 \mathrm{~mm}$

Fig. 5 Distribution of circumferential stress $\sigma_{\mathrm{p}}$ and longitudinal stress $\sigma_{\mathrm{l}}$ in cross section at $z=z_{\mathrm{R}}=115.0 \mathrm{~mm}(P=7.0 \mathrm{~mm}$, $r_{\mathrm{R}}=45.6 \mathrm{~mm}$, simulation result).

shows the distribution of the $\rho^{-1}$ in the circumferential direction at the reduced portion under the conditions with $P=7.0 \mathrm{~mm}$ and $L=16.0 \mathrm{~mm}$. In the figure, the $\rho^{-1}$ is shown under the conditions of $r_{\mathrm{R}}=45.6 \mathrm{~mm}, z_{\mathrm{R}}=110.0$ $115.0 \mathrm{~mm}$, and $\alpha=36^{\circ}-126^{\circ}$. The $\rho^{-1}$ decreases sharply to $0.001 \mathrm{~mm}^{-1}$ at $\alpha=54^{\circ}$. Focusing on the angular position of $\theta=54^{\circ}$, the $\rho^{-1}$ is $0.025 \mathrm{~mm}^{-1}$ at $\alpha=36^{\circ}$, whereas the $\rho^{-1}$ sharply decreases to $0.001 \mathrm{~mm}^{-1}$ at $\alpha=54^{\circ}$. At $\alpha \geq 90^{\circ}$, the $\rho^{-1}$ takes an almost constant value of $0.010 \mathrm{~mm}^{-1}$ and does not reach the value of $0.022 \mathrm{~mm}^{-1}$. Namely, when the $L$ is large, the unbending deformation is induced rapidly as a result of contact with the roller. The curvature becomes uneven in the circumferential direction. The results of further simulation indicate that a dent is generated at $\theta=54^{\circ}$, which engenders wrinkling. The value of $\Delta r$ at the necked portion of the tube obtained under these forming conditions is $14.3 \mathrm{~mm}$, which is an indication of forming defect.

For a more detailed analysis of the mechanisms underlying excess deformation of unbending and bending, we discuss stresses calculated in the simulation. Figure 5 shows the region of contact between the roller and the tube, the distributions of the circumferential stress $\sigma_{\mathrm{p}}$ and longitudinal stress $\sigma_{1}$ in the contact region. The values of stress in the outer, middle, and inner layers are shown in each figure. The abscissa, $\theta-\alpha$, represents the relative position between the tube and the roller, for example, $\theta-\alpha=0^{\circ}$ means the position where the roller is directly above the tube. Figure 5(a) shows the stress distribution in the cross section at $z=z_{\mathrm{R}}=$ $115.0 \mathrm{~mm}$ for $r=r_{\mathrm{R}}=45.6 \mathrm{~mm}$ after necking with $P=7.0$ $\mathrm{mm}$ and $L=6.0 \mathrm{~mm}$. The $\sigma_{\mathrm{p}}$ in the inner layer is larger than that in the outer layer when $\theta-\alpha$ is approximately $4^{\circ}$, which indicates the occurrence of deformation attributable to unbending by the decrease of the curvature in Fig. 4(a). Moreover, $\sigma_{\mathrm{p}}$ in the inner layer becomes smaller than that in the outer layer at $\theta-\alpha<-2^{\circ}$, which corresponds to the bending deformation by the increased curvature. The value of $\sigma_{\mathrm{p}}$ in the middle layer is compressive, which indicates that the tube deforms due to bending and unbending under circumferential compressive stress. The stress distribution under $L=16.0 \mathrm{~mm}$ and $P=7.0 \mathrm{~mm}$, is presented in Fig. 5(b). The relations among $\sigma_{\mathrm{p}}$ in the inner, outer, and middle layers are similar to those under $L=6.0 \mathrm{~mm}$. 


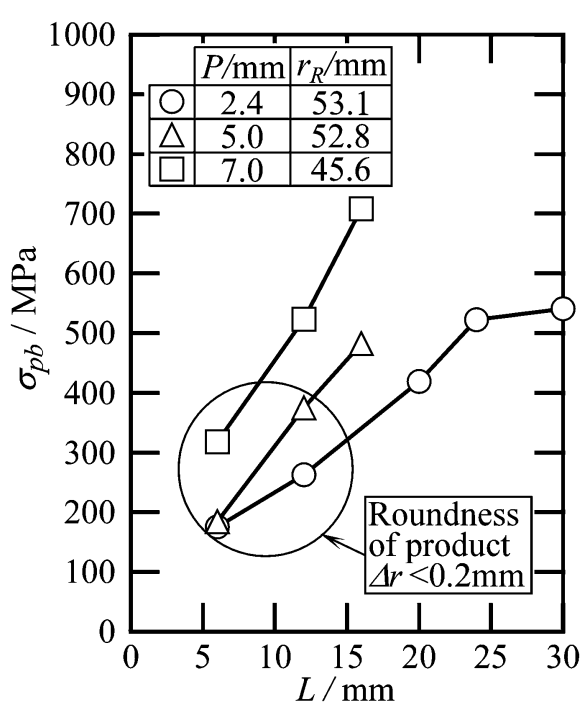

Fig. 6 Effect of forming conditions on maximum $\sigma_{\mathrm{pb}}$ of the difference of outer $\sigma_{\mathrm{p}}$ and inner $\sigma_{\mathrm{p}}$ in contact area shown in Fig. $5\left(z_{\mathrm{R}}=115.0 \mathrm{~mm}\right.$, simulation result)

However, the difference in $\sigma_{\mathrm{p}}$ between the inner and outer layers when $\theta-\alpha=4^{\circ}$ under $L=16.0 \mathrm{~mm}$ is much greater than that under $L=6.0 \mathrm{~mm}$. The difference in $\sigma_{\mathrm{p}}$ between the inner and outer layers was then calculated. The maximum value while the tube is in contact with the roller is denoted as $\sigma_{\mathrm{pb}}$. Figure 6 shows the effects of the forming conditions on $\sigma_{\mathrm{pb}}$. The $\sigma_{\mathrm{pb}}$ increases concomitantly with increasing $P$ and $L$. In this study, for the $\sigma_{\mathrm{pb}}$ in the circled Fig. 6 , the $\Delta r$ of the necked portion is smaller than $0.2 \mathrm{~mm}$. It was confirmed experimentally and by simulation that the formed products have high dimensional accuracy.

Consequently, the mechanisms underlying the deformation of the tube and the occurrence of a wrinkling can be explained as follows: When the pitch and axial feed rate of the roller are large, the tube is subjected to unbending and the curvature suddenly decreases in the circumferential direction by the contact with the rollers. After the tube is separated from the roller, the tube is subjected to bending and excessive localized forming, which causes the uneven curvature in the circumferential direction, thereby resulting in wrinkling.

The right sides of Figs. 5(a) and 5(b) respectively show the distributions of longitudinal stress $\sigma_{1}$ under $L=6.0 \mathrm{~mm}$ and $L=16.0$, respectively. The absolute value of $\sigma_{1}$ in the middle layer becomes less than $100 \mathrm{MPa}$, which is small in comparison with the absolute value of $\sigma_{\mathrm{p}}$ in the middle layer.

\subsection{Relation between roundness of the tube and number of contacts with rollers}

To shorten the forming time, it is preferred that the forming pitch in the axial and radial directions be large. The number of contacts with the rollers $n_{\mathrm{c}}$ between the tube in an arbitrary $r-z$ plane and the rollers is defined ${ }^{11)}$ as the parameter used to determine the optimum forming conditions that result in a short forming time and high dimensional accuracy. For this study, the $n_{\mathrm{c}}$ is calculated using eq. (1) with the length of the roller locus $F_{k}$ and $B_{k} \quad(k=$ $1,2, \cdots N, F)$ in the $r-z$ plane, as shown in Fig. 1(c).

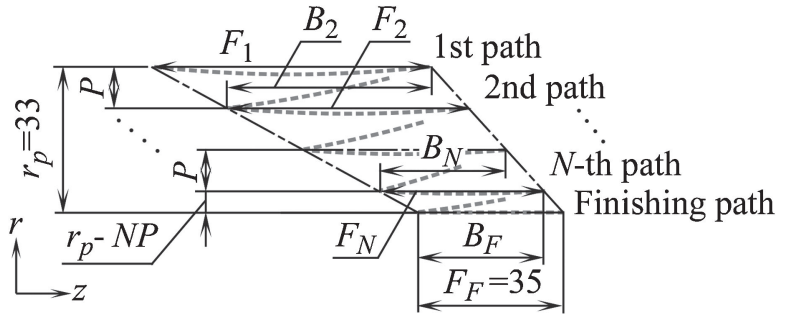

Fig. 7 Notations of locus length shown in Fig. 1(c).

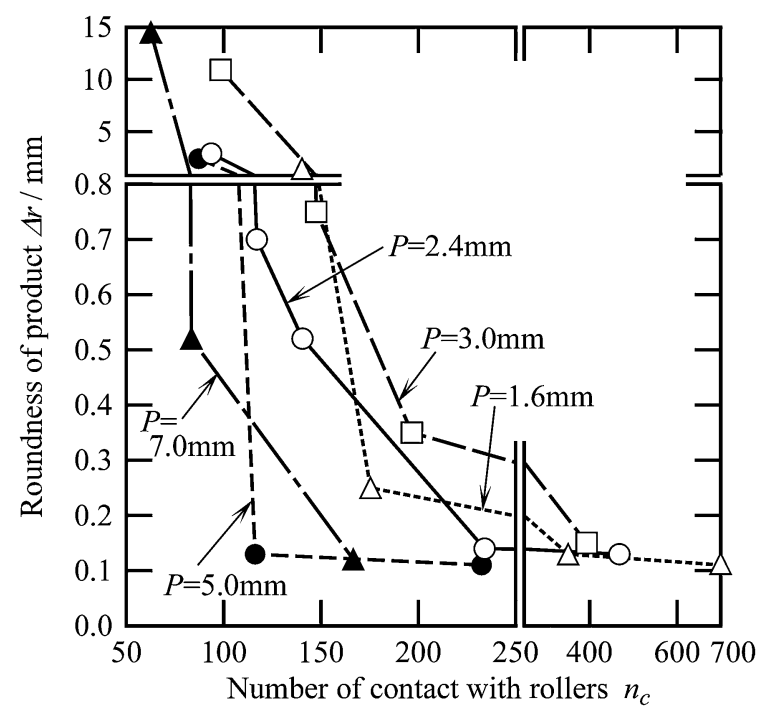

Fig. 8 Effect of radial pitch $P$ on relationship between roundness of product $\Delta r$ and number of contact with rollers $n_{\mathrm{c}}\left(N_{\mathrm{R}}=2\right.$, experimental results).

$$
\begin{aligned}
n_{\mathrm{c}}= & \frac{N_{\mathrm{R}}}{L}\left[\left\{\operatorname{int}\left(\frac{r_{\mathrm{p}}}{P}\right)+1\right\}\left(\frac{F_{1}+F_{N}}{2}\right)\right. \\
& \left.+\operatorname{int}\left(\frac{r_{\mathrm{p}}}{P}\right)\left(\frac{B_{2}+B_{N}}{2}\right)+\left(F_{F}+B_{F}\right)\right]
\end{aligned}
$$

The variables in eq. (1) represent the lengths shown in Fig. 7. The length of the roller locus can be given as an arithmetic progression. The first term in the square bracket in eq. (1) represents the length of the forward pass from the first to the $N$ th path. The second term represents the length of the backward pass from the second to the $N$ th path, and the third term represents the length of the final path. As is obvious from eq. (1), the $n_{\mathrm{c}}$ is proportional to the $N_{\mathrm{R}}$, and inversely proportional to the $L$. Furthermore, as the $P$ increases, the $n_{\mathrm{c}}$ decreases in almost inversely proportional manner.

Figure 8 shows effects of the $P$ on the relationship between the $\Delta r$ and the $n_{\mathrm{c}}$ with $N_{\mathrm{R}}=2$. For any $P \mathrm{~s}$, the $\Delta r$ increases sharply the $n_{\mathrm{c}}$ decreases. In regard to the $\Delta r$ at $n_{\mathrm{c}}=150$, it is apparent that $\Delta r \geq 0.5 \mathrm{~mm}$ when $P \leq 3.0 \mathrm{~mm}$, whereas $\Delta r \leq 0.2 \mathrm{~mm}$ when $P \geq 5.0 \mathrm{~mm}$. Therefore, it follows that $P \geq 5.0 \mathrm{~mm}$ is the optimum condition for decreasing $n_{\mathrm{c}}$ and obtaining small roundness among the forming conditions examined in this study.

Figure 9 shows the effect of the $N_{\mathrm{R}}$ on the relationship between the $\Delta r$ and the $n_{\mathrm{c}}$ with $P=2.4 \mathrm{~mm}$. For any $N_{\mathrm{R}} \mathrm{s}$, changes in the $\Delta r$ with respect to the $n_{\mathrm{c}}$ show similar trends. 


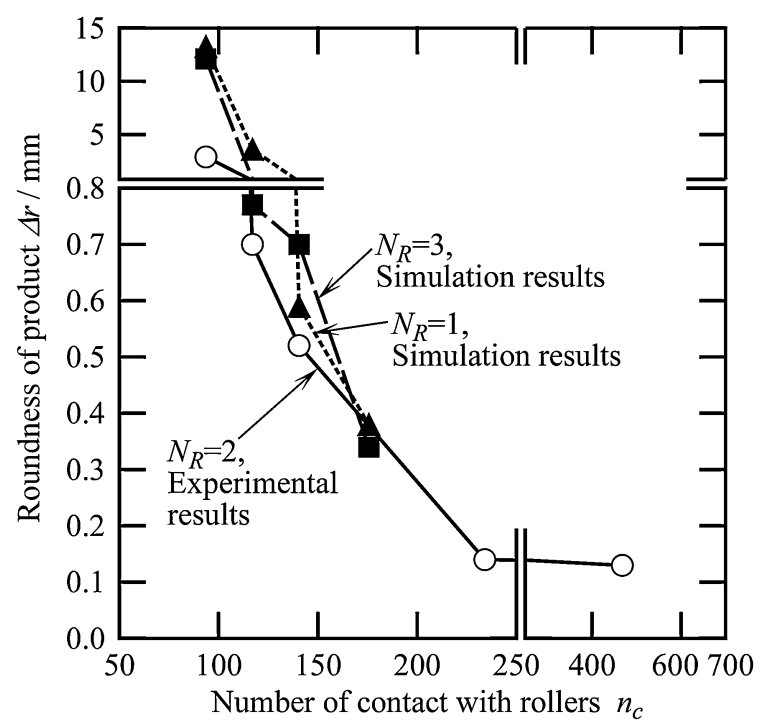

Fig. 9 Effect of number of rollers $N_{\mathrm{R}}$ on relationship between roundness of product $\Delta r$ and number of contact with rollers $n_{\mathrm{c}}(P=2.4 \mathrm{~mm})$.

In the case of the condition that the $P$ is constant, the $\Delta r$ is dependent on the $n_{\mathrm{c}}$. Therefore, tubes with equivalent roundness can be formed even if the $N_{\mathrm{R}}$ is increased. Accordingly, the $L$ can be increased to shorten the forming time.

\section{Conclusion}

(1) In the necking process, the tubes gradually shrink and become formed through repetitive unbending and bending, inducing the decrease and increase of the curvature, due to the contact with and separation from the roller under a circumferential compressive force by rollers.
(2) When the axial or radial forming pitch of the rollers is large, the outside of the tube is subjected to considerable compressive stress in the circumferential direction because of the contact with the rollers. Simultaneously, the inside of the tube is subjected to considerable tensile stress in the circumferential direction. At that point, the tube is subjected to excessive localized unbending and forming, causing the uneven curvature in the circumferential direction, thereby resulting in wrinkling.

(3) A method was proposed for calculating the number of contacts occurring with the roller in the $r-z$ plane to derive the relationship between the number of contacts and the roundness of the necked portion of the tube. The optimum forming conditions were determined from the results to achieve rapid forming and high dimensional accuracy easily.

\section{REFERENCES}

1) M. Hayama: Advances in Rotary Forming Processes, kindai Henshu Ltd., Tokyo, (1992) pp. 241-249. (in Japanese)

2) M. Murata and R. Muta: J. JSTP 42 (2001) 124-128. (in Japanese)

3) A. Danno: J. JSTP 43 (2002) 1023. (in Japanese)

4) J. Yao and M. Murata: J. Mater. Process. Technol. 166 (2005) 405-410.

5) A. Andou, K. Sakamoto, S. Karino and K. Hara: Proc. 2007 Japanese Spring Conf. for the Technology of Plasticity, Nagoya, (2007) pp. 185186. (in Japanese)

6) Q. X. Xia, X. Q. Cheng, Y. Hu and F. Ruan: Int. J. Mech. Sci. 48 (2006) 726-735.

7) Q. X. Xia, Sh. W. Xie, Y. L. Huo and F. Ruan: J. Mater. Process. Technol. 206 (2008) 500-508.

8) S. Kihara, H. Mizumoto, H. Ishida and Y. Iio: Proc. 2006 Japanese Spring Conf. for the Technology of Plasticity, Tokyo, (2006) pp. 217218. (in Japanese)

9) S. Kihara, H. Mizumoto, H. Ishida, Y. Iio and Y. Nakata: Proc. 57th Japanese Joint Conf. for the Technology of Plasticity, Takaoka, (2006) pp. 367-368. (in Japanese)

10) H. Takizawa and T. Kimura: J. JSTP 40 (1999) 987-991. (in Japanese)

11) H. Takizawa and T. Kimura: J. JSTP 40 (1999) 343-347. (in Japanese) 